\section{Memory metabolism}

Effector and memory T cells show differences in their metabolic states and use of energy sources. In Immunity, Pearce and colleagues explore how $\mathrm{CD}^{+} \mathrm{T}$ cells switch their metabolic state to become memory cells. Unlike glycolytic effector cells, memory $\mathrm{CD}^{+} \mathrm{T}$ cells use oxidative phosphorylation to generate ATP. Stimulation with IL-15 triggers more mitochondrial spare respiratory capacity (reflected by more oxygen consumption), but stimulation with IL-2 does not. These changes are correlated with more biogenesis of mitochondria, as more mitochondria are found in memory cells than in naive or effector CD8+ $\mathrm{T}$ cells. IL-15 induces upregulation of CPT1, a rate-limiting enzyme that allows the cells to use fatty acids as an energy source. Modulation of CPT1 alters the generation of memory cells in vitro and, notably, in vivo in response to infection with Listeria monocytogenes. Thus, IL-15 contributes to memory development by regulating mitochondrial abundance and function.

$L A D$ Immunity 36, 1-11 (2012)

\section{Regulating neutrophil homeostasis}

Neutrophils are the most abundant cells of the immune system, and their population therefore requires careful regulation. Bensinger and colleagues in the Journal of Clinical Investigation identify a pivotal action of the liver X receptors (LXRs) in neutrophil homeostasis. Knocking out LXR $\alpha$ and LXR $\beta$ leads to many more neutrophils but has no apparent intrinsic effect on the neutrophils themselves. Senescent neutrophils are normally cleared by macrophages, which elicits a negative feedback signal against granulopoiesis. Accordingly, the authors find that engulfment of apoptotic neutrophils by macrophages activates LXR signaling, which then downregulates their expression of interleukin 23 (IL-23). This decrease in IL-23 in turn diminishes the abundance of $\mathrm{IL}-17^{+}$cells in the spleen and, critically, the amount of the granulopoietic cytokine G-CSF. Phagocyte LXRs therefore are important both in mediating the tolerogenic signals to phagocytes and in the process of neutrophil homeostasis.

J. Clin. Invest. (12 January 2012) doi:10.1172/JCI58393

\section{A tale of two bugs}

Invasive infection with Salmonella is a common complication in people with malaria. In Nature Medicine, Riley and colleagues show that loss of resistance to Salmonella is due to the hemolytic release of heme and the subsequent induction of heme oxygenase (HO-1), which is essential for tolerance to the cytotoxic effects of free heme during malaria. Progressive release of free heme and HO-1 impairs maturation of the oxidative-burst ability of granulocytes in the bone marrow and mobilizes functionally immature granulocytes in the peripheral circulation, which can phagocytose but cannot kill bacteria and thus provide a niche for the replication and dissemination of Salmonella. Inhibition of HO-1 restores normal development of the oxidative burst in maturing bone marrow granulocytes and resistance to Salmonella. These results indicate that resistance and tolerance mechanisms are pathogen specific and can diminish resistance to another pathogen.

Nat. Med. 18, 120-127 (2012)

\section{Innate immunity activators}

The growth factor GM-CSF is a pleiotropic cytokine with activatory properties for innate leukocytes and is believed to be produced mostly by non-hematopoietic cells and macrophages. In Science, Rauch et al. show that phenotypically and functional distinct B cells, called 'innate response-activator' (IRA) B cells, are the largest GM-CSF-producing population in models of sepsis and bacterial infection. IRA B cells develop from peritoneal B-1 cells that migrate to the spleen and differentiate after signaling via Toll-like receptor 4 and autocrine GM-CSF signaling. IRA B cells have a surface marker and transcriptional profile distinct from that of follicular, marginal-zone or transitional B cell subtypes and spontaneously secrete immunoglobulin M and IL-3 but do not produce IL-10, IL-6 or tumor-necrosis factor (TNF). Deletion of IRA B cell-specific activity impairs bacterial clearance, elicits a cytokine storm and precipitates septic shock.

Science (12 January 2012) doi:10.1126/science.1215173

\section{Linking p65 to RPS3}

After exposure to inflammatory signals, activation of the transcription factor NF-kB is needed to upregulate expression of antiapoptotic genes that confer cell survival. In Molecular Cell, Sen et al. show that TNF transiently upregulates expression of cystathionine $\gamma$-lysase (CSE), producing hydrogen sulfide $\left(\mathrm{H}_{2} \mathrm{~S}\right)$ that then modifies Cys38 of NF-kB (subunit p65) to create a hydropersulfide (-SSH). Sulfhydration of Cys38 increases the DNAbinding activity of $\mathrm{p} 65$ and expression of the antiapoptotic factor $\mathrm{Bcl}-\mathrm{x}_{\mathrm{L}}$, the deubiquitinase A20, the ubiquitin ligase cIAP2 and the apoptosis inhibitor XIAP. Higher expression of genes encoding prosurvival molecules is linked to more association of p65 with its cofactor, ribosome protein S3 (RPS3). At later time points, p65 is nitrosylated at Cys38, which blunts both its ability to interact with RPS 3 and its DNA-binding activity. Inhibition of CSE, substitution of p65 Cys38 or knockdown of RPS3 in TNF-stimulated cells leads to lower expression of genes encoding prosurvival molecules and more cell apoptosis. These findings demonstrate the importance of the interaction of p65 with RPS3 for cell survival and provide insight into how their association is regulated.

\title{
Delineating interferon population genetics
}

Interferons are a diverse family of cytokines with critical roles in immunity. In the Journal of Experimental Medicine, Quintana-Murci and colleagues use population genetics to delineate the relative importance of members of the interferon family in human immunity. Interferons are categorized into three families according to the receptors they bind and their functional and signaling properties: type I (INF- $\alpha 1-I N F-\alpha 13$, INF$\beta$, INF- $\varepsilon$, INF- $\kappa$ and INF- $\omega$ ), type II (INF- $\gamma$ ) and type III (INF- $\lambda 1-I N F-$ $\lambda 3$ ). By looking at the mutation frequency of genes encoding the various interferons and how this relates to different geographical distributions (Africa, Europe and Asia), they are able to gauge the contribution to host survival. Notably, IFN- $\gamma$ and, to a lesser extent, IFN- $\alpha 6$, IFN- $\alpha 8$, IFN- $\alpha 13$ and IFN- $\alpha 14$ show strong purifying positive selection in all populations, which suggests critical nonredundant functions in immunity to infection. Furthermore, strong positive selection is also seen for type III interferons, but only in Eurasian populations, probably as an adaptive response to specific pathogen exposure in this geographical locale. J. Exp. Med. (12 January 2012) doi:10.1084/jem.20111680 